\title{
A numerical investigation of heat transfer cardiac output measurements
}

\author{
P. FOTHERINGHAM $\dagger$, A. R. GOURLAY $\dagger, S . M C K E E * \ddagger$ and S. ANDREWS \\ $\dagger$ Centre for Mathematical and Computational Science in Medicine, University of Glasgow, Glasgow G12 8QQ, UK \\ ‡Department of Mathematics, University of Strathclyde, 26 Richmond Street, Glasgow G1 1XH, UK \\ ๆ AorTech International plc., Strathclyde Business Park, Bellshill, Lanarkshire ML4 3NJ, UK \\ (Received 18 December 2002; revised 10 December 2003; in final form 31 March 2005)
}

\begin{abstract}
Measurement of cardiac output is often investigated using a technique based on hot-film anemometry. Here, we discuss a modification to hot-film anemometry, which involves a cylindrical heating element mounted flush on the surface of a typical Swan-Ganz catheter. In contrast to traditional thermodilution, the method discussed here has the potential to allow continuous monitoring of cardiac output.

This paper demonstrates that there is a simple approximate relationship between the power input to the device to maintain a temperature of one degree above blood heat and cardiac output. Since, the heat transfer and the fluid flow decouple, a numerical model of the heat transfer of a cylindrical catheter (with heating element) sitting concentrically within a rigid cylindrical artery is developed. Numerical results were obtained for a wide selection of flow profiles, including experimental data. The results indicate that the cardiac output/power input relationship is extremely robust with respect to flow profile and system parameter variation.
\end{abstract}

Keywords: Cardiac output; Pulsatility; Hot-film anemometry; Annulus; Thermodilution

\section{Introduction}

Cardiac output (CO), is simply the volume of blood passing through the heart in a given time. Typically, it is measured in litres per minute and can be thought of as the product of stroke rate and volume for the chambers of the heart. For an average patient, the $\mathrm{CO}$ should be around 4 or $51 \mathrm{~min}^{-1}$ but can drop to as low as 2 in critical situations. It is an important diagnostic factor [1,2] during high risk surgery, particularly transplantation, where it is used in conjunction with blood pressure to assess the performance of the heart. It is also used post-operatively in intensive care.

Traditionally, clinicians have used thermodilution to measure $\mathrm{CO}$ [1]. This involves inserting a catheter into the subclavian or jugular vein at the neck and feeding it through until it reaches the right atrium. The catheter is then guided into the right ventricle and subsequently the pulmonary artery by inflation of a balloon at the catheter tip. Once the balloon has carried the catheter into position an injection of cold saline ( $\sim$ few degrees centigrade) is injected through the catheter and emerges from a port which should reside in the right atrium. A thermistor is located downstream prior to the tip in the pulmonary artery and measures the new temperature of the mixture at this point. The $\mathrm{CO}$ can then be calculated based on a modification of the Stewart-Hamilton equations, which involve various quantities of the blood and the saline e.g. densities, pre/post injection temperatures, etc. The Stewart-Hamilton equations were originally used to predict $\mathrm{CO}$ from dye indicator methods [3] but suffer from many assumptions [4].

There are many disadvantages of thermodilution. For each $\mathrm{CO}$ measurement required, one has to carry out a separate injection. To obtain an accurate value, it is often the case that about three or four measurements have to be taken consecutively and the average value is used. Measurements are therefore time consuming and far from ideal if constant monitoring is required. Available nursing time is very often the limiting factor in the use of thermodilution in post-operative case. Repeated injections of saline also present an infection risk and the possibility of fluid imbalance in patients thereby limiting the amount of measurements that can be taken. Recent work by [5] has

*Corresponding author. Email: smck@maths.strath.ac.uk. Tel.: +44-141-548-3671. Fax: +44-141-552-8657

Journal of Theoretical Medicine

ISSN 1027-3662 print/ISSN 1607-8578 online (c) 2005 Taylor \& Francis

http://www.tandf.co.uk/journals

DOI: $10.1080 / 10273660500158712$ 
shown that a single injection of controlled duration can give at least the same accuracy as the traditional averaging method and so some of the problems above are alleviated. However, the calculation of $\mathrm{CO}$ from thermodilution also relies on a number of assumptions, one of which is that the injectate enters as a single bolus and then mixes thoroughly with the blood. To ensure the former depends greatly on the skill of the person administering the injection and can vary between different clinicians.

One of the biggest disadvantages of this method, however, is in the discrete nature of the results obtained. Furthermore, an assumption of the Stewart-Hamilton equations is that the blood flow is steady and so changes in $\mathrm{CO}$ cannot be accurately measured. A technique, which could allow continuous monitoring of $\mathrm{CO}$ would be an enormous improvement provided all other factors are equal i.e. accuracy, cost, etc. There has been considerable work attempting to adapt thermodilution so that the technique might provide continuous readings. For example, it is possible to use a heat exchanger where the saline is part of a closed loop and does not mix with the blood as described by [6]. This technique allows continual repeated measurements but these are still roughly half a minute apart. The initial results also showed that the technique suffered from a bias of around $-0.51 \mathrm{~min}^{-1}$ compared to conventional thermodilution measurements i.e. across a range of cardiac outputs it underestimated $\mathrm{CO}$, on average, by $0.51 \mathrm{~min}^{-1}$.

Alternatively, some techniques heat the blood directly with a heating element e.g. [7]. For one such method including a review of previous work see [8]. These methods can be limited by the resolution of the downstream temperature measurements when compared to the background thermal noise since the blood can only be heated minimally before cell damage occurs. More recently, this limitation has been overcome using improved signal processing techniques and varying the position and duration of the heat pulses; however, this approach suffers from a delayed response to changes in CO e.g. [9-11]. There has also been disagreement in the literature over the comparison of this technique with conventional thermodilution. [12] report a bias as low as $0.031 \mathrm{~min}^{-1}$ and [13] quote one as high as $-0.81 \mathrm{~min}^{-1}$.

In contrast to thermodilution, methods utilizing hot-film anemometry (HFA) ([14]), can provide continuous CO measurements with little or no intervention required after the initial catheter insertion. The principle of HFA is that the power required to maintain the temperature of the element at a fixed difference to the ambient blood is related to the speed of the blood flowing past it. The catheter itself is guided into position in an identical way to the thermodilution case. Early studies ([15-17]) used a double conical quartz probe where the film is located at the extreme tip of the catheter and oriented transversely to the flow in the traditional spirit of HFA. Since the crosssectional area of the artery is unknown, the probe is calibrated in-situ via an initial thermodilution injection. [18] reported good correlation between this approach and traditional thermodilution measurements in a laboratory set-up although they did note that recalibration was required if the catheter changed position.

A factor that is particularly important in this approach is the profile of the blood velocity within a blood vessel whose average diameter is of the order of a few centimetres. This is further complicated by the fact that the artery radius changes throughout the heart cycle. Details of measured flow profiles are discussed later in this paper. However, in summary, the flow can be quite complicated and can exhibit nonaxisymmetric behaviour and reversing flow. This is compounded by the catheter itself, which affects the flow by its presence within the chambers of the heart through to the artery itself. The flow behaviour is clearly patient dependent and so it is important that the technique be insensitive to parameter changes if it is to be of any real value.

In this work, we wish to study a hot-film anemometer with a configuration which differs from the previously discussed work in two ways. Firstly, the heating element is located prior to the tip, typically 7 or $8 \mathrm{~cm}$ from it, and therefore, resides in the pulmonary artery. Secondly, the film itself takes the form of a cylindrical heating element mounted flush with the length of the catheter, see figure 1. The reason for studying this particular device was through a data fitting request from an industrial partner looking for the correct functional form for the relationship between $\mathrm{CO}$ and the input power to the device. After early discussions the question was reposed as, "Can we use the underlying conservation equations to deduce a simple relationship between $\mathrm{CO}$ and power input?" Once this relationship was established, the next question was, "Is this relationship robust with respect to the assumptions made and the parameters of the system?" Primarily, how important is neglecting the viscous boundary layer? Is the relationship robust with respect to pulsatility? What is the effect of varying the pulmonary artery size, the heart beat and the amplitude of the heart beat. Does varying the velocity profiles make a significant difference to the $\mathrm{CO} /$ power relationship?

An outline of the rest of the paper is as follows. First we review some known results and present a simple mathematical model in two dimensional Cartesian coordinates; this is then employed to study the effect of parameter variation. Next we consider the flow profiles generated by periodic pressure gradients in a cylindrical geometry.

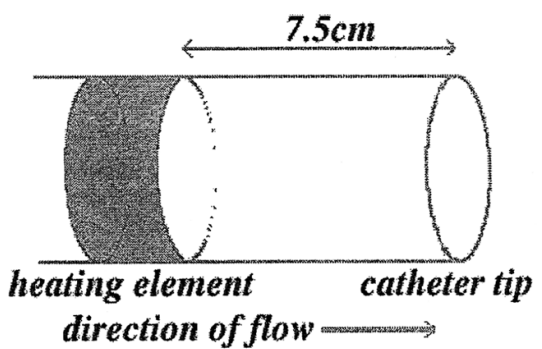

Figure 1. The position and nature of the heating element on the catheter. 
A numerical model for this cylindrical geometry is then developed in which various velocity profiles (some based on experimental results) are used to convect heat away from the device. The paper concludes with a discussion of these results.

\section{Elementary heat transfer models}

\subsection{Notation}

We shall denote fluid velocity by the vector $\mathbf{u}$ with Cartesian components $u_{x}, u_{y}$ and $u_{z}$ and the temperature field by $T$. The power input to the heating element (per unit length in the $x$-direction) will be represented by $Q$. The thermal diffusivity of the fluid will be denoted by $\kappa$ and is related to the thermal conductivity $k$ through $k=\kappa c \rho$ where $c$ is the specific heat at constant pressure and $\rho$ is the fluid density. The kinematic viscosity of the fluid will be denoted by $\nu$ and is related to the dynamic viscosity $\mu$ by $\mu=\rho \nu$. The Prandtl number Pr is defined to be the ratio of kinematic viscosity to thermal diffusivity i.e. $\nu / \kappa$.

\subsection{Plane wall cartesian models}

Heat transfer from a plane wall to a liquid has been studied extensively in the early literature, for example, [19-21, 34]. In summary, these boundary layer analyses treated incompressible Newtonian fluids in semi-infinite domains. They gave rise to scaling laws, which suggested that the power required to maintain a fixed wall temperature against a fixed ambient fluid temperature varied as the square root of the mainstream velocity.

An imposed flow analysis by [22] took into account a finite length $L$ of heating element but, like the analyses above, assumed that the radius of the artery was infinite, or at any rate considerably greater than $L$, and imposed the shear flow

$$
u_{x}=0, \quad u_{y}=0, \quad u_{z}=S y
$$

where $S$ is the shear rate. Here $z$ is the direction of the flow. The fluid exists in the region $y>0$, with the heating element at $y=0$ having an assumed infinite extent in $x$. The solution of this system leads to a power per unit perpendicular length of

$$
\frac{3^{1 / 3}}{2 \Gamma(4 / 3)} k\left(T_{1}-T_{0}\right)\left(\frac{S L^{2}}{\kappa}\right)^{1 / 3} .
$$

where $T_{1}$ and $T_{0}$ are the wall and ambient fluid temperatures, respectively and where $\Gamma(\nu)$ is the gamma function. In a boundary layer model such as those mentioned above the wall shear rate can be shown to vary with the mainstream velocity as $U_{0}^{3 / 2}$. This confirms that the power required to maintain the element at a constant temperature would vary with the square root of the mainstream velocity.

Pedley then went on to discuss a time varying shear rate of the form $S(1+\xi \sin \mathrm{t})$. The solution for the required power output was in the form of a sum of terms, the first of which represented the "quasi-steady" part i.e. a sinusoidal variation about the steady value. The regime in which the quasi-steady solution dominates the sum is in the low frequency and short $L$ limit. With $\xi=0.5$ and a $1 \mathrm{~cm}$ long heating element some rough estimates show that the next term in the sum can be ignored if the angular frequency is not bigger than $10^{-1} \mathrm{~s}^{-1}$ i.e. 1 beat per minute (bpm). Alternatively, for a typical heart rate of $80 \mathrm{bpm}$, the quasisteady approximation is only valid for a heating element of length shorter than $10^{-3} \mathrm{~cm}$. The question is then still open as to what effect a typical heart rate will have on the mean power requirement as compared to a steady flow. Even if the quasi-steady solution is not an appropriate approximation to the time varying solution it may still be the case that the period-averaged values are close to the steady values.

\subsection{Slug flow heat transfer}

The data obtained from the device that was supplied for the initial curve fitting exercise did indeed follow a square root dependence but not quite as described above (see figure 2). A better fit was obtained if a constant term was added i.e.

$$
Q=C_{1} U_{0}^{1 / 2}+C_{2}
$$

where $C_{1}$ and $C_{2}$ are constants. A model, which yields such a result can be obtained very easily. Continuing the

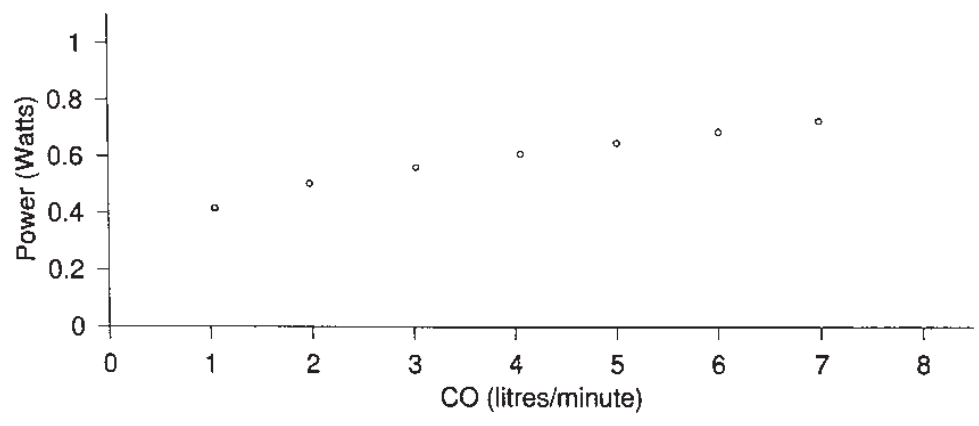

Figure 2. Original experimental data supplied by AORTECH. 
theme of a plane wall geometry it is possible to obtain an analytical solution using the extremely simple velocity profile of slug flow

$$
u_{x}=0, \quad u_{y}=0, \quad u_{z}=U_{0}
$$

where $U_{0}$ is just a constant. A top boundary is also added at $y=b$ to represent an artery wall. This relaxes the noslip fluid boundary condition in favour of a stress-free condition. At first sight this would appear to be inappropriate since it assumes the thermal boundary layer is larger than the viscous boundary layer which is being ignored. Since $\operatorname{Pr}>1$ for blood, this assumption would normally make the model inappropriate; however, there are reasons not to dismiss it out of hand. Firstly, it fits the actual data from the device better than the models previously discussed. Secondly, the previous models assumed that $b \gg L$ when, in fact, $b \sim O(L)$ whereas, this simple model crucially does not. Finally, it must be recalled that in reality the artery walls are not rigid. It is not clear how the viscous or indeed thermal boundary layers develop when the walls are moving.

Using the length of the heating element, $L$, as a typical length scale in the $z$ direction and the radius of the artery, $b$, as a typical length scale in the $y$ direction, the temperature equation may be written as

$$
\frac{\partial \Theta}{\partial z}=\lambda \frac{\partial^{2} \Theta}{\partial y^{2}}, \quad \lambda=\frac{\kappa L}{U_{0} b^{2}}
$$

where the non-dimensional temperature $\Theta$ is defined through $T=T_{0}+\left(T_{1}-T_{0}\right) \Theta$.

The boundary conditions imposed are

$$
\begin{aligned}
& \Theta=0 \quad \text { at } z=0, \\
& \Theta=1 \quad \text { at } y=0, \\
& \Theta=0 \quad \text { at } y=1 .
\end{aligned}
$$

Laplace transforms can be used to derive the solution

$$
\begin{aligned}
\Theta(y, z) & =1-y+\frac{2}{\pi} \sum_{n=1}^{\infty} \frac{(-1)^{n}}{n} \\
& \times \sin ((1-y) n \pi) \exp \left(-n^{2} \pi^{2} \lambda z\right) .
\end{aligned}
$$

The power (in dimensional terms) lost in the positive $y$ direction per unit length in the $x$ direction through the surface $y=0$ is then

$$
\begin{aligned}
Q= & k\left(T_{1}-T_{0}\right) \\
& \times \frac{L}{b}\left[1+\frac{2}{\pi^{2} \lambda} \sum_{n=1}^{\infty} \frac{1}{n^{2}}\left(1-\exp \left(-n^{2} \pi^{2} \lambda\right)\right)\right] .
\end{aligned}
$$

It is clearly desirable to try to find a closed form description of the summation in equation (2). Estimates for $\kappa, L$ and $b$ of $10^{-7} \mathrm{~m}^{2} \mathrm{~s}^{-1}, 10^{-2} \mathrm{~m}$ and $10^{-2} \mathrm{~m}$, respectively give $\lambda \sim O\left(10^{-5} U_{0}^{-1}\right)$. Typical values for $\mathrm{CO}$ are $1-71 \mathrm{~min}^{-1}$ which translates to values of $U_{0}$ around $3-25 \mathrm{~cm}^{-1} \mathrm{~s}$ i.e. $\lambda \sim 4 \times 10^{-5}-3.3 \times 10^{-4}$.
Thus, we see that these estimates give values of $\lambda \ll 1$ and clearly as $\lambda \rightarrow 0$ it can be shown that the sum is sandwiched between the same limit, namely $\left(\lambda \pi^{3}\right)^{1 / 2}$ and so one would expect that behaviour of the sum for small but finite $\lambda$ would be approximately $\left(\lambda \pi^{3}\right)^{1 / 2}$.

Returning to equation (2), we see that the power $Q$ may be accurately approximated by

$$
Q \sim k\left(T_{1}-T_{0}\right) \frac{L}{b}\left[1+\frac{2 b}{(\pi \kappa L)^{1 / 2}} U_{0}^{1 / 2}\right] .
$$

Of course, one must remember that the power quoted above is still per unit length in the $x$ direction. In other words, there exists constants $C_{1}$ and $C_{2}$ such that

$$
Q \sim C_{1} U_{0}^{1 / 2}+C_{2}
$$

agreeing with the experimental evidence.

\section{Pulmonary artery flow profiles}

The main purpose of this work is to investigate the relationship between $\mathrm{CO}$ and the required heating power in the relevant cylindrical geometry to see if the square root behaviour persists and whether the pulsatility of the blood flow affects the mean power value significantly.

In particular, our model will be a cylindrical annulus since the catheter will be present within the artery. To simplify matters, we will assume that the catheter sits concentrically within the artery where both are assumed to be perfectly cylindrical and rigid. We also assume that there is no movement of the catheter within the artery, which of course is unlikely to be true. [23] have shown that the position of a catheter in the pulmonary artery tends to be roughly the same at the start and finish of a heart cycle and also at the point of peak cross-sectional area of the artery. In between times, however, they found that there was random motion with the catheter sometimes coming very close to the artery walls. Since the motion appears to be periodic, it is hoped that this effect would average itself out over the course of each heart cycle. Furthermore, the randomness of the motion is likely to be averaged out over several heart cycles.

We shall ignore thermal buoyancy effects since the nondimensional parameter comparing the buoyancy force to the inertial force is small. If we take our timescale to be $f^{-1}$ where $f$ is the frequency of the heart cycle then this parameter, in the most general 3D sense, can be stated as

$$
g \beta\left(T_{1}-T_{0}\right) /\left(f U_{0}\right)
$$

where $g$ is the gravitational acceleration and $\beta$ is the volumetric coefficient of thermal expansion of blood (typically around $4 \times 10^{-4} \mathrm{~K}^{-1}$ ). With typical values of $T_{1}-T_{0} \sim 1 \mathrm{~K}, f \sim 1.5 \mathrm{~s}^{-1}$ and $U_{0} \sim 0.1 \mathrm{~ms}^{-1}$, the ratio of buoyancy to inertial terms is of the order $3 \times 10^{-2}$ so that the momentum and heat equations can be treated as uncoupled. We can therefore establish our flow profiles at 
the outset and insert them into the heat equation at the time of computation.

This then raises the question of what the correct profiles should be. For example, is fully developed pipe flow appropriate or not? Various techniques such as MRI and traditional HFA have been used to investigate the flow profiles in the pulmonary artery. [24] studied the porcine pulmonary artery and reported a flat profile at early systolic acceleration and at late systolic deceleration with a non-axisymmetric peaked profile in between, that is, with a peak skewed towards the artery wall. This type of behaviour was also noted by [25] in 12 human volunteers. The latter also noted some localized backflow during diastole. Despite the presence of non-axisymmetric peaks in the profile, we have chosen for simplicity to assume an axisymmetric profile. Although, an accurate velocity profile would be desirable the purpose of this work is to test the effect of the periodicity of the flow and not its structure. However, we shall study both the flat slug flow profile and the fully developed flow (with an axisymmetric peak) as well as a combination of these to test sensitivity of periodicity to the profile. The combination profile is chosen to exhibit similar spatial and temporal behaviour to the Sloth et al. and Sømod et al. profiles albeit with the peak being axisymmetric rather than localized. In addition to being axisymmetric, the numerical code is also limited to non-reversing flows.

Although blood is a non-Newtonian fluid, it will be modelled here as an incompressible Newtonian fluid for the purposes of calculating the fully developed profiles. According to [26] the transition from non-Newtonian behaviour occurs as the shear rate increases above $20 \mathrm{~s}^{-1}$ and becomes fully Newtonian past $100 \mathrm{~s}^{-1}$. With $U_{0}=0.1-0.2 \mathrm{~ms}^{-1}$ as typical peak velocities about halfway from the catheter, a rough estimate of the shear would be $\sim 20-40 \mathrm{~s}^{-1}$ placing us in the transition regime. It should be noted though that the work of [27] suggests that blood behaves as a Newtonian fluid when the Womersley number (Wo) exceeds unity. We define the Wo later but note that the values we obtain are typically in the range $6-8$. In what follows we shall take the kinematic viscosity of blood $\nu$ to be $4 \times 10^{-6} \mathrm{~m}^{2} \mathrm{~s}^{-1}$ and the density $\rho$ to be $1055 \mathrm{~kg}^{-3}$.

\subsection{Notation and non-dimensionalization}

We shall denote a point in space in cylindrical polar coordinate by $(s, \phi, z)$. Let the $z$ axis be the axis of the cylindrical annulus which has inner radius $s=a$ and outer radius $s=b$. We shall non-dimensionalize the radial coordinate $s$ by the outer radius $b$ so that the two radii are now $a / b$ and 1 . In practice, we shall always take $a=0.0025 \mathrm{~m}$ and $b=0.0125 \mathrm{~m}$.

The $z$ direction will be non-dimensionalized by $L$ and the velocity by $U_{0}$. In the case of slug flow, $U_{0}$ will be the amplitude of the steady part of the profile whereas, in the fully developed flow, $U_{0}$ shall be the maximum velocity in the steady part of the generalized Poiseuille profile discussed next.

\subsection{Poiseuille flow in a cylindrical annulus}

Assume that the flow can be written as

$$
\mathbf{u}(s, \phi, z)=u(s) \mathrm{e}_{z} .
$$

If we substitute this into the Navier-Stokes equations along with a constant pressure gradient, $\Delta P$, we are left to solve the following problem,

$$
\frac{1}{s} \frac{\mathrm{d}}{\mathrm{d} s}\left(s \frac{\mathrm{d} u}{\mathrm{~d} s}\right)=\frac{b^{2} \Delta P}{\rho \nu U_{0}}=\gamma,
$$

say with

$$
u(a / b)=0, \quad u(1)=0 .
$$

This yields

$$
u(s)=u_{P}(s)=\frac{\gamma}{4}\left[s^{2}-1-\left(\frac{a^{2}}{b^{2}}-1\right) \frac{\ln s}{\ln (a / b)}\right] .
$$

One might note that this solution is a special case of the solution obtained by [28] which is for the general case of parallel cylinders which are not concentric. It would be interesting to extend the current work to use these types of solution but we shall not discuss this any further here.

The maximum velocity is attained when

$$
s=\left(\frac{\left(a^{2} / b^{2}\right)-1}{2 \ln (a / b)}\right)
$$

and so defining this as $u=1$ (i.e. $U_{0}$ in dimensional terms) determines $\gamma$ as

$$
\gamma^{-1}=\frac{1}{4}\left\{\frac{\left(a^{2} / b^{2}\right)-1}{2 \ln (a / b)}\left[1-\ln \left(\frac{\left(a^{2} / b^{2}\right)-1}{2 \ln (a / b)}\right)\right]-1\right\} \text {. }
$$

In terms of dimensional $\mathrm{CO}\left(\mathrm{m}^{3} \mathrm{~s}^{-1}\right)$ we have

$$
\mathrm{CO}=2 \pi b^{2} U_{0} \int_{a}^{b} u(s) s \mathrm{~d} s
$$

which, upon substitution of equation (3), gives

$$
\mathrm{CO}=2 \pi U_{0} \frac{\gamma}{16}\left(a^{2}-b^{2}\right)\left(\frac{a^{2}}{b^{2}}+1+\frac{\left(1-\left(a^{2} / b^{2}\right)\right.}{\ln (a / b)}\right) .
$$

So, for a given $\mathrm{CO}$, we can obtain the actual value of $U_{0}$ by first converting from $1 \mathrm{~min}^{-1}$ into $\mathrm{m}^{3} \mathrm{~s}^{-1}$ by multiplying by $(1 / 6) \times 10^{-4}$ and eliminate $\gamma$ using equation (4).

\subsection{Womersley flow in a cylindrical annulus}

We now consider a periodic pressure gradient i.e.

$$
\frac{\partial P}{\partial z}=\Delta P \delta_{W} \cos (2 \pi t)
$$

where we have non-dimensionalized time $t$ with $1 / f$ where $f$ is the frequency of oscillation corresponding to the heart 
rate. We have ignored the higher harmonics since the Navier-Stokes equations are linear in $u$ under our assumptions and we are only interested in the first harmonic for the velocity. The parameter $\delta_{W}$ simply represents the relative amplitude to that of a Poiseuille flow driven by $\Delta P$. As before, we assume laminar flow so that the Navier-Stokes equations reduce to

$$
\frac{\partial u}{\partial t}=\Gamma \cos (2 \pi f t)+\frac{1}{W o^{2}} \frac{1}{s} \frac{\partial}{\partial s}\left(s \frac{\partial}{\partial s}\right) u
$$

where

$$
\Gamma=-\Delta P \delta_{W} /\left(\rho f U_{0}\right), \quad W o=b(f / \nu)^{1 / 2}
$$

Wo is known as the Womersley number (see, e.g. [29],[30]). As before the boundary conditions assume no slip. Since the flow is assumed to be fully developed we shall seek an equilibrated solution sharing the periodicity of the pressure gradient, i.e.

$$
\begin{aligned}
u(s, t) & =u_{W}(s, t) \\
& =U_{1}(s) \cos (2 \pi t)+U_{2}(s) \sin (2 \pi t) .
\end{aligned}
$$

Substituting this into equation (5) and equating similar time dependencies gives two coupled second order ordinary differential equations for $U_{1}$ and $U_{2}$ subject to the boundary conditions

$$
U_{1}(a / b)=U_{1}(1)=0, \quad U_{2}(a / b)=U_{2}(1)=0 .
$$

After some complex algebra, one obtains a general solution of the form

$$
\begin{gathered}
U_{1}(s)=C_{1} J_{0}(\text { Woos })+C_{2} Y_{0}(\text { Woos }) \\
+C_{3} I_{0}(\text { Woos })+C_{4} K_{0}(\text { Woos }) \\
U_{2}(s)=\Gamma / 2 \pi-i C_{1} J_{0}(\text { Woos })-i C_{2} Y_{0}(\text { Woos }) \\
+i C_{3} I_{0}(\text { Woos })+i C_{4} K_{0}(\text { Woos })
\end{gathered}
$$

where $\alpha=\pi^{1 / 2}(1+i)\left(i=(-1)^{1 / 2}\right)$ and $J_{0}$ and $Y_{0}$ are the zero order Bessel functions of the first and second kind, respectively and $I_{0}$ and $K_{0}$ are the equivalent modified functions. In applying the boundary conditions we shall denote

$$
J_{a}=J_{0}(W o \alpha(a / b)), \quad J_{b}=J_{0}(W o \alpha)
$$

with similar definitions for the other types of Bessel functions. The no-slip boundary conditions supply the constants

$$
\begin{array}{ll}
C_{1}=\frac{-i \Gamma}{4 \pi} \frac{Y_{b}-Y_{a}}{J_{a} Y_{b}-J_{b} Y_{a}}, & C_{2}=\frac{-i \Gamma}{4 \pi} \frac{J_{a}-J_{b}}{J_{a} Y_{b}-J_{b} Y_{a}} \\
C_{3}=\frac{-i \Gamma}{4 \pi} \frac{K_{a}-K_{b}}{I_{a} K_{b}-I_{b} K_{a}}, & C_{4}=\frac{-i \Gamma}{4 \pi} \frac{I_{b}-I_{a}}{I_{a} K_{b}-I_{b} K_{a}} .
\end{array}
$$

Plots of $U_{1}$ and $U_{2}$ along with the steady profile $u_{P}$ can be found in figure 3 where each quantity is normalized to one. The value of the Wo has been chosen to be 6.25 to reflect a heart rate of $60 \mathrm{bpm}$.

For both the steady and periodic parts, we have assumed that the form of flow we have imposed is stable. The Reynolds number $R e$, defined as $U_{0} b / \nu$, is 500 if we take $U_{0}=0.2, b=0.01$ which, as we shall see, will be typical values in what follows. [31] experimentally derived a relationship between the critical $R e$ and Wo for straight pipes. This relationship is

$$
R e_{\text {crit }}=169(W o)^{0.87}(S t)^{-0.27}
$$

where the Strouhal number $S t$ is $f b / U_{\text {osc }}$ and $U_{\text {osc }}$ is the peak velocity of the purely oscillatory component. Again substituting typical values gives $S t \sim 1$ (based on $U_{\text {osc }} \sim 0.2 U_{0}$ for $\delta_{W}=6.0$ ) and $W o$ as low as 5. This results in a critical $R e$ of 685 and suggests that the flow will be non-turbulent.

\subsection{Simulated velocity profiles}

We shall use three different velocity profiles throughout the calculations that follow. Firstly, we shall impose a pulsatile flat (slug) profile of the form

$$
u_{S}=1+\delta_{S} \cos (2 \pi t)
$$

for various values of $\delta_{s}$. Secondly, we shall use the fully developed profile obtained from the superposition of equations (3) and (6), the modified Poiseuille and

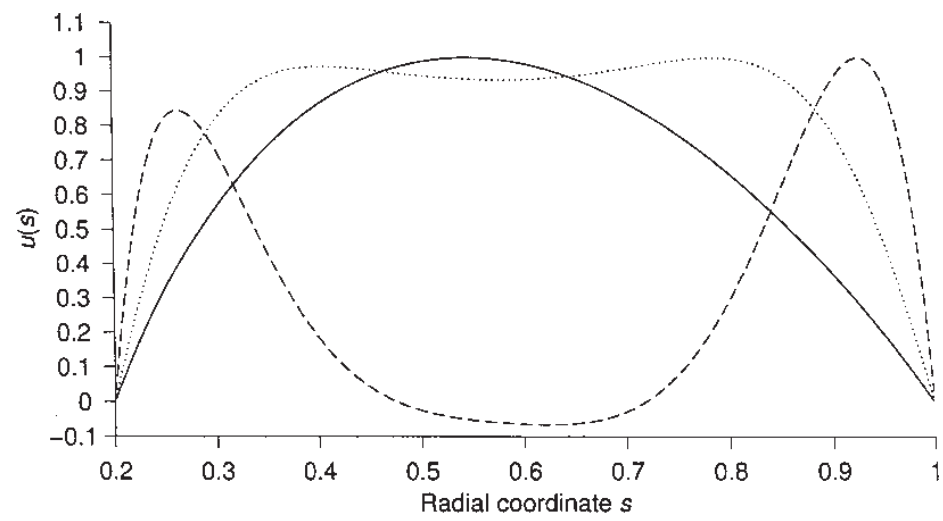

Figure 3. Flow profiles corresponding to $u_{P}$ (solid), $U_{1}$ (dashed) and $U_{2}$ (dotted) all normalized to one. The unsteady profiles are shown for $W_{0}=6.25$ corresponding to a heart rate of around $60 \mathrm{bpm}$. 
Womersley flows respectively, i.e.

$$
u_{F D}=u_{P}+u_{W} .
$$

Recall that this is driven by

$$
\frac{\partial P}{\partial z}=\Delta P\left(1+\delta_{W} \cos (2 \pi t)\right)
$$

For $\delta_{W}=1$, the relative amplitude of $\mathrm{u}_{W}$ to $u_{P}$ is very small and of little interest. To make $u_{W}$ comparable to $u_{P}$ without producing any localized backflow, a value of $\delta_{W}=6$ was chosen. Four equally spaced snapshots of this profile can be seen in figure 4. Lastly, we shall use a combination of the previous two profiles in an attempt to create a more realistic profile matching those of [25]. The steady part will be flat and the systolic peak will be modelled by the function $U_{2}$ from the modified Womersley solution during the first half of the heart cycle. This takes the form

$$
u_{M}=\left\{\begin{array}{cc}
\delta_{M}+\bar{U}_{2} \sin (2 \pi t) & 0 \leq(t-n) \bmod 1<0.5 \\
\delta_{M} & 0.5 \leq(t-n) \bmod 1<1
\end{array},\right.
$$

$$
n \in \mathbb{Z}
$$

where $\bar{U}_{2}$ is just $U_{2}$ normalized such that the maximum amplitude is unity since we are no longer controlling the flow through the pressure gradient parameter $\delta_{W}$. It is noted that this bears a striking resemblance to the time dependence of the pulmonary artery velocities reported by [32] and so it may not be too simplistic.

In each of the three cases above, the heat transfer results obtained will be compared with those from an equivalent steady model whose velocity is defined by

$\int_{0}^{1} u(s, t) \mathrm{d} t$.

The first two cases simply return the steady part of the profile due to the sinusoidal nature of the unsteady part. However, the mixed profile returns

$\delta_{M}+\bar{U}(s) / \pi$.

Note that this steady solution actually depends on the Wo through $\bar{U}_{2}$. This means that two unsteady solutions, corresponding to different heart rates, have to be compared against two different equivalent steady solutions. All this means is that even though there is no actual time dependence, the steady solutions will have a heart rate associated with them purely to determine the profile. In addition, the non-sinusoidal nature of the unsteady part means that it contributes to the cardiac output. For the mixed profile we therefore have to relate the specified dimensional $\mathrm{CO}$ to $U_{0}$ by

$$
\mathrm{CO}=2 \pi U_{0} \delta_{M}\left(b^{2}-a^{2}\right)+2 b^{2} U_{0} \int_{a / b}^{1} \bar{U}(s) s \mathrm{~d} s
$$

where the integral can be evaluated analytically by noting that

$$
\begin{aligned}
\int U_{2}(s) s \mathrm{~d} s= & \frac{\Gamma}{4 \pi} s^{2}-\frac{i C_{1}}{W o \alpha} s J_{1}(W o \alpha s)-\frac{i C_{2}}{W o \alpha} s Y_{1}(W o \alpha s) \\
& +\frac{i C_{3}}{W o \alpha} s I_{1}(W o \alpha s)-\frac{i C_{4}}{W o \alpha} s K_{1}(W o \alpha s)
\end{aligned}
$$

\section{The temperature equation and its numerical solution in a cylindrical annulus}

The non-dimensional equation governing the temperature of the blood near the catheter heating element for the given blood flow rate $u(s, t) \mathrm{e}_{z}$ is

$$
\sigma \frac{\partial \theta}{\partial t}+u(s, t) \frac{\partial \theta}{\partial z}=\frac{\lambda}{s} \frac{\partial}{\partial s}\left(s \frac{\partial \theta}{\partial s}\right) .
$$

Here, the non-dimensional temperature is given by

$$
\theta=\left(T-T_{0}\right) /\left(T_{1}-T_{0}\right)
$$

and

$$
\sigma=f L / U_{0} \quad \text { and } \quad \lambda=K L /\left(U_{0} b^{2}\right) .
$$

Equation (11) is to be solved on the domain

$$
(a / b) \leq s \leq 1, \quad 0 \leq z \leq 1, \quad t \geq 0,
$$

subject to the initial condition

$$
\theta(s, z, 0)=0
$$

and the boundary conditions

$$
\theta(s, 0, t)=0, \quad \theta((a / b), z, t)=h(z), \quad \theta(1, z, t)=0,
$$

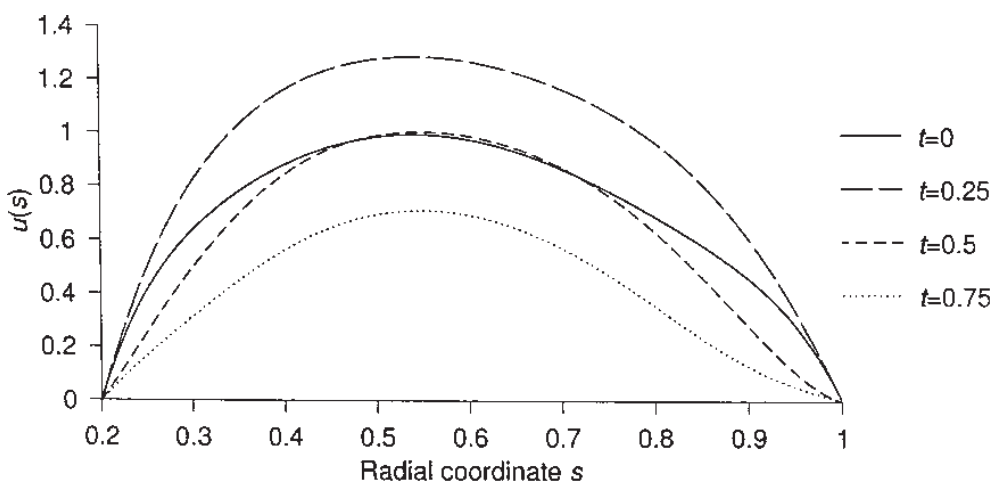

Figure 4. Four equally spaced snapshots throughout one period of the fully developed flow profile defined by $\delta_{M}=6$. 
where the function $h(z)$ is defined as

$h(z)= \begin{cases}z^{2}\left[3-2 z / z_{1}\right] / z_{1}^{2}, & 0 \leq z<z_{1} \\ 1, & z_{1} \leq z<1-z_{1} \\ (z-1)^{2}\left[3+2(z-1) / z_{1}\right] / z_{1}^{2}, & 1-z_{1} \leq z \leq 1\end{cases}$

and is designed to resolve the discontinuity at $(s, t)=(a / b, 0)$. The value of $z_{1}$ was fixed at $10^{-2}$ throughout.

This problem was then solved by finite differences using the method due to [33]. The power required by the heating element was evaluated using the trapezoidal rule. The radial derivative of the temperature in the integrand was approximated by second order differences.

Extensive grid refinement was used to determine mesh spacings that would produce results correct to $0.1 \%$. This resulted in the choice $\Delta s=10^{-4}, \Delta z=10^{-3}$ and $\Delta t=10^{-3}$ and these values were used throughout. In fact, the code was originally written so that it would operate on a stretched grid; however, given the relatively modest CPU time required for the uniform grid, the benefits of employing it were not significant.

\section{Results}

\subsection{Parameter variations}

Before presenting the results themselves we shall briefly outline the values used in the simulations. Recall that there are three parameters to vary, one for each of the models chosen. Firstly, $\delta_{S}$ represents the amplitude of the oscillatory component of the slug flow velocity profile. Then there is $\delta_{W}$ which is the amplitude of the oscillatory component of the pressure driving the fully developed flow. Finally, there is $\delta_{M}$, which represents the amplitude of the steady component of the mixed velocity profile.

For the slug flow model $\delta_{S}$ takes the values $0.5,0.8$ and 0.95 at a heart rate of $80 \mathrm{bpm}$ initially. Heart rates of 60 and $100 \mathrm{bpm}$ are then subsequently tested for all three values. The fully developed model uses only $\delta_{W}=6$ and is investigated at heart rates of 60,80 and $100 \mathrm{bpm}$. The mixed profile is run with $\delta_{M}=0.2$ again at heart rates of 60,80 and $100 \mathrm{bpm}$; values of 0.1 and 0.3 are then investigated at $80 \mathrm{bpm}$.

\subsection{Pulsatile slug flow profile}

We use the profile given by equation (8) and set $\delta_{S}=0.5$ initially. Starting with $\mathrm{CO}$ of $21 \mathrm{~min}^{-1}$ and a heart rate of $80 \mathrm{bpm}$ (Wo $=7.217$ ) the temperature equation (11) was stepped forward in time from an initial condition where $\Theta$ was zero everywhere. Onset of periodicity was checked at each timestep by comparing $\theta(t)$ and $\theta(t-1)$ values at three spatial locations to check that each agreed to better than $10^{-4} \%$. The locations chosen were along the line $s=(a / b)+(1-(a / b)) / 100$ at $z=\Delta z, 0.5$ and $1-\Delta z$. After just over two heart cycles, the solution had equilibrated to the same periodicity as the flow. CO was incremented in steps of 0.4 and the results can be seen as the solid line of figure 5 where the power required to be input to the heating element is plotted against the square root of the cardiac output as measured in $1 \mathrm{~min}^{-1}$. Results for a purely steady slug flow are superimposed as circles. It is immediately apparent that both trends are linear and so we have confirmed that the dependence of power on the square root of $\mathrm{CO}$ holds in the more realistic cylindrical geometry. The dataset corresponding to the unsteady flow has a slightly lower gradient than the steady dataset with close agreement at the lower end. Changing the heart rate to either $60 \mathrm{bpm}(W o=6.25)$ or $100 \mathrm{bpm}(W o=8.069)$ has no noticeable effect on this result.

If the above sequence of runs are repeated with $\delta_{S}=0.8$ and 0.95 we obtain the behaviour shown by the dashed and dotted lines in figure 5. Again the change of heart rate makes no difference although the separation of the steady and unsteady trends is a little more pronounced at these higher values of $\delta_{S}$. For instance, at a CO of $7.61 \mathrm{~min}^{-1}$ with $\delta_{S}=0.95$, the steady state model overestimates the power loss by approximately $7 \%$.

To ensure that the numerical method was indeed giving correct results, a convergence check was performed with a $\mathrm{CO}$ value of $7.61 \mathrm{~min}^{-1}$ and with $\delta_{S}=0.8$. The number of

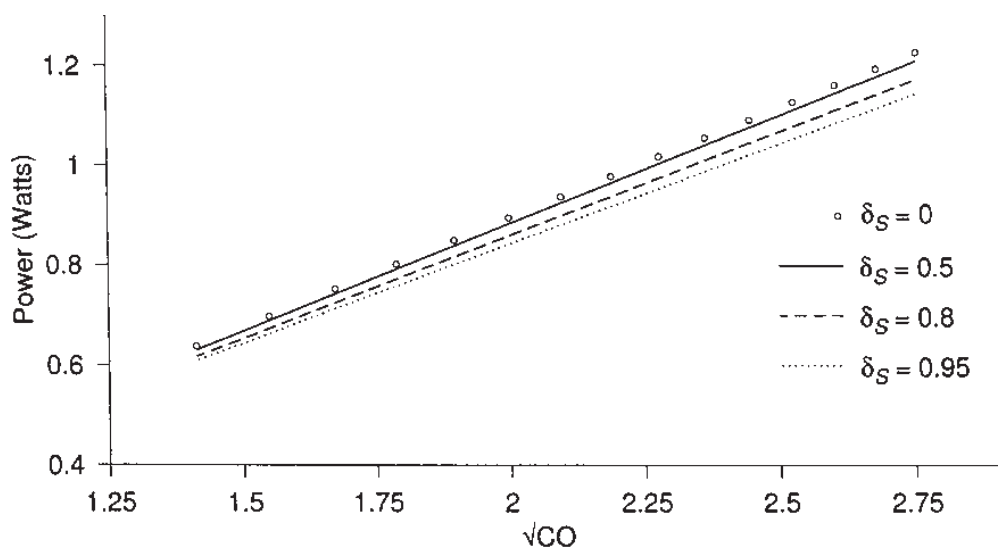

Figure 5. Comparison of power input vs. square root of cardiac output for a periodic slug flow profile with various values of $\delta_{S}$. The heart rate is $80 \mathrm{bpm}$ throughout. 


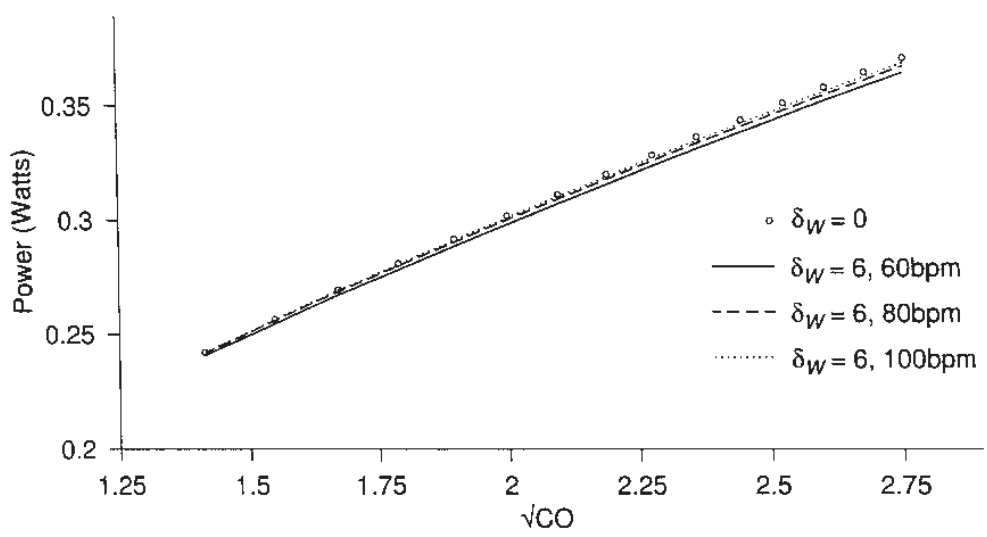

Figure 6. Comparison of power input vs. square root of cardiac output for the fully developed flow profile. The steady case is denoted by circles and the unsteady cases, defined by $\delta_{M}=6.0$, are denoted by the various lines.

grid points in the $s$-direction was doubled producing a change of less than $0.1 \%$ in the power. A similar size of change was noted when the number of grid points in the $z$-direction was doubled. This confirms that the increasing separation of the two models with increasing $\mathrm{CO}$ is a real effect.

\subsection{Fully developed flow profile}

For the fully developed flow profile, the change of heart rate may have more impact since the shape of the profile changes with the Wo. As previously discussed, we chose $\delta_{W}=6.0$ and considered heart rates of 60,80 and $100 \mathrm{bpm}$.

The results of stepping through a sequence of $\mathrm{CO}$ values are shown in figure 6 . The first point to notice is the significant difference in the amount of power required by the heating element. Compared to the slug flow, there is, on average, only a third of the power required. This is due to the lower velocities present near the element since this profile satisfies a no-slip boundary condition. The second point to notice is that the relationship between the power drawn by the element and the square root of the cardiac output is not exactly linear. There is a noticeable curve to both the steady and unsteady datasets, which is more apparent for lower cardiac outputs. Both of these points show that calibration would be highly dependent on the velocity profile. This is not all that surprising since the hot-film approach measures velocities rather than volumetric flow rates and this drawback of the technique has been noted many times before, see for example $[1,16]$.

As we expected, changing the heart rate has a more noticeable effect than for the slug flow, where there was no change whatsoever. The effect increases with decreasing heart rate in a non-linear fashion as shown by the separation of the solid and dashed lines to that of the dashed and dotted lines in figure 5. At a $\mathrm{CO}$ of $7.61 \mathrm{~min}^{-1}$ the difference between steady and average unsteady power at $60 \mathrm{bpm}$ is just under $2 \%$ which is still clinically acceptable.

\subsection{Mixed flow profile}

We shall initially set $\delta_{M}=0.2$ and look at a heart rate of $80 \mathrm{bpm}$. The results are shown in figure 7 . In contrast to the previous two velocity profiles, the degree of separation of steady and unsteady trends is not as obviously dependent on the size of the cardiac output. In fact, the unsteady model underestimates the power by $54 \mathrm{~mW}$ at a $\mathrm{CO}$ of $21 \mathrm{~min}^{-1}$ and by $60 \mathrm{~mW}$ at a $\mathrm{CO}$ of $81 \mathrm{~min}^{-1}$ showing that there is a slight increase in discrepancy with increasing CO.

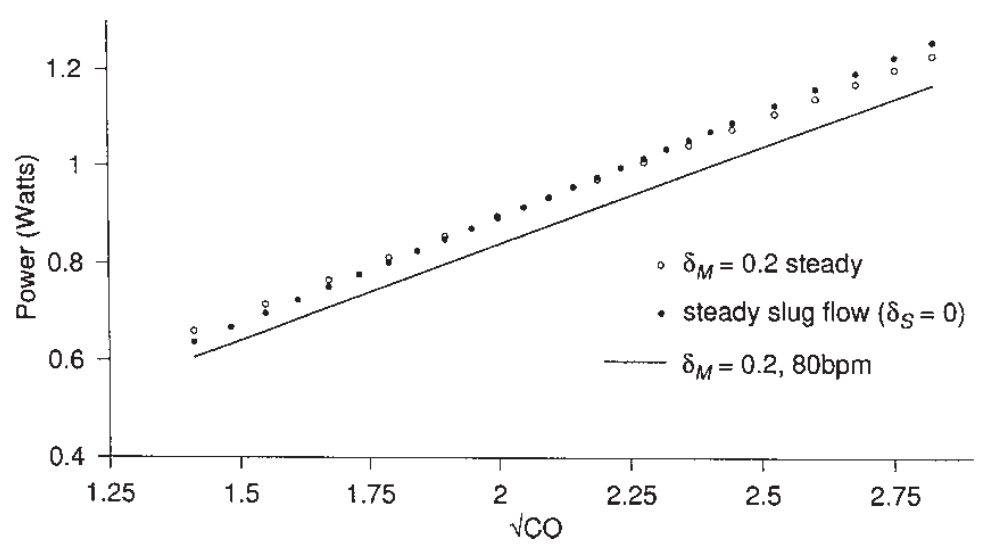

Figure 7. Comparison of power input vs. square root of cardiac output for the mixed flow profile. The cases considered are the unsteady solution, defined by $\delta_{M}=0.2$ and a heart rate of $80 \mathrm{bpm}$, the corresponding steady solution and finally the steady slug flow profile solution. 


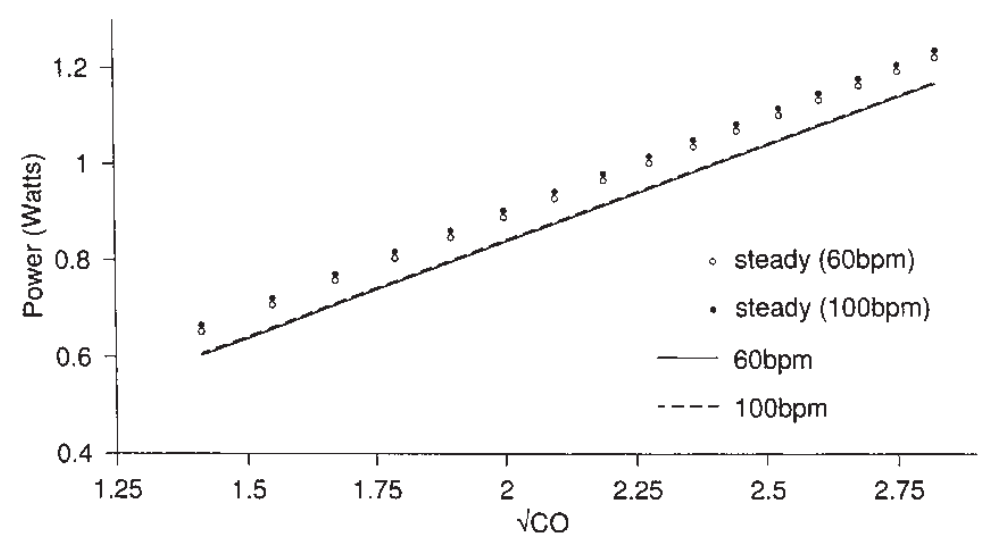

Figure 8. Comparison of power input vs. square root of cardiac output for two mixed flow profiles with differing heart rates. Both profiles are defined by $\delta_{M}=0.2$.

One might be tempted to seek a better comparison with a purely flat profile for the steady solution since this represents the steady part of the pulsatile flow. The filled circles in figure 7 indicate the steady slug flow results that have already been presented. The agreement is not better and again the discrepancy exhibits an increasing size towards the upper end of the scale.

Changing the heart rate makes little difference to the results as shown in figure 8 . What is apparent here is that the steady solution seems to be more affected than the pulsatile one resulting in a bigger gap for higher heart rates. This makes sense since in the limit of vanishing heart rate, the function $\bar{U}_{2} \rightarrow 0$. This means that both the unsteady and equivalent steady solution will converge to the slug flow solution. The effect is, however, clearly small for the realistic range of heart rates such as those simulated here.

Finally, we take a look at the effect of varying $\delta_{M}$ at a fixed heart rate of $80 \mathrm{bpm}$ in figure 9. The trends for each value of $\delta_{M}$ shows different gradients although the equivalent steady solution always essentially mirrors that of the unsteady solution. Again this is not surprising since in the limit $\delta_{M} \rightarrow \infty$, we are again moving towards the slug flow solution which we know from figure 7 has a steeper gradient than the mixed profile model.

More interesting is the variation in the discrepancy between the steady and pulsatile models. As $\delta_{M}$ is decreased the gap increases. Table 1 shows the value of $U_{0}$ for three values of $\delta_{M}$ and three values of the cardiac output. For $\delta_{M}=0.1$ the values of $V_{0}$ are too high and so this is an unrealistic choice. The values of $U_{0}$ for $\delta_{M}=0.2$ are more realistic and those for $\delta_{M}=0.3$ agree reasonably well with the peak velocities reported in both $[25,32]$. By the time $\delta_{M}$ has increased to 0.3 , the discrepancy is not as pronounced with the power being overestimated by roughly $40 \mathrm{~mW}$ by the steady model. Alternatively, one can say that, given a power reading, the steady model underestimates the $\mathrm{CO}$ by $\sim 0.41 \mathrm{~min}^{-1}$.

\subsection{Response to changes in arterial diameter}

Since the system is being solved numerically, it is straightforward to test the effect of varying the diameter of either the catheter or the artery in which it resides. There are two approaches: either hold the $\mathrm{CO}$ constant when changing the geometry or hold the peak steady velocity $U_{0}$ constant. Since, the catheter has a fixed diameter we choose to vary the artery radius using both approaches outlined above.

The slug flow situation is by far the simplest. Here, the CO varies directly with $U_{0}$ with the constant of proportionality being equal to the cross-sectional area of the annulus. For a constant $\mathrm{CO}$, the velocity therefore varies inversely with the $\sqrt{b^{2}-a^{2}}$. For the case of constant velocity, the power will

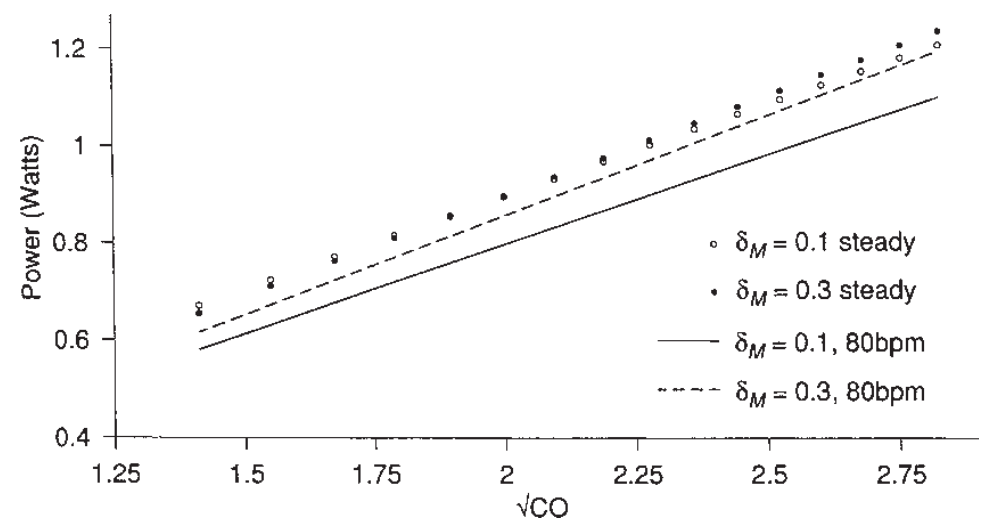

Figure 9. Comparison of power input vs. square root of cardiac output for two mixed flow profiles defined by differing values of $\delta_{M}$. The heart rate is $80 \mathrm{bpm}$ throughout. 
Table 1. Values of $U_{0}$ in $\mathrm{ms}^{-1}$ for various $\delta_{M}$ and CO.

\begin{tabular}{lccl}
\hline $\mathrm{CO}(1 / \mathrm{min})$ & 2.0 & 4.0 & 6.0 \\
\hline$\delta_{M}=0.1$ & 0.49 & 0.97 & 1.5 \\
$\delta_{M}=0.2$ & 0.29 & 0.58 & 0.86 \\
$\delta_{M}=0.3$ & 0.20 & 0.41 & 0.61 \\
\hline
\end{tabular}

be independent of artery radius provided it is not near the edge of the thermal boundary layer of course. This was indeed confirmed by the numerics.

The fully developed flow is more interesting since the profile is not constant across the thermal boundary layer. For a constant $\mathrm{CO}$, the power drawn varies inversely with the $\sqrt{b^{2}-a^{2}}$ as shown by the solid line in figure 10 . This is not obvious since it is not clear that the amplitude of the velocity profile within the thermal boundary layer varies inversely as $\sqrt{b^{2}-a^{2}}$. For a constant $U_{0}$, the power is not independent of $b$ since the steady velocity profile will have a larger gradient at the boundary for smaller artery radii. This means that relatively more power will be drawn for smaller artery radii.

The mixed flow also shows the same behaviour as the previous two profiles when constant $\mathrm{CO}$ is maintained throughout the radii variation. This is shown by the dashed line in figure 10 . For a constant $U_{0}$, the power is once again independent of $b$ and so the steady part of the profile must be dominating the behaviour here.

\subsection{Response time to changes in $\mathrm{CO}$}

So far we have timestepped the temperature field in the presence of a pulsatile flow and allowed the solution to equilibrate over time from some arbitrary initial condition. There is nothing to prevent us from using the solution at one cardiac output and using it to move to that at another. Of course, we are completely ignoring the dynamics of how the flow would evolve from one state to the other and instead simply changing instantaneously between the two.
However, this should give us a rough idea of the timescale on which changes in $\mathrm{CO}$ could be picked up, i.e. how long it takes for the period averaged power to settle into a new equilibrated value.

Using the mixed profile model at $\delta_{M}=0.2$ and a heart rate of $80 \mathrm{bpm}$ the initial condition was set to be the equilibrated solution at a $\mathrm{CO}$ of $21 \mathrm{~min}^{-1}$. The value of $\mathrm{CO}$ for the run was then set to be $61 \mathrm{~min}^{-1}$ and the system stepped forward in time. It took just under four and a half heartbeats to settle into periodicity (using our prescribed tolerance which could easily be relaxed). A similar time was noted for the reverse change from 6 to $21 \mathrm{~min}^{-1}$. This is more than adequate for the requirements of clinical practice.

\section{Conclusions}

We have shown that the results obtained using pulsatile models of heat transfer in our simple cylindrical annular model differ from those using equivalent steady models. The difference was found to be largely independent of the heart rate for realistic ranges of this parameter $(60-100 \mathrm{bpm})$. Using a flat velocity profile, the discrepancy between the steady and the pulsatile models was found to increase with increasing $\mathrm{CO}$ whereas this was not observed with the mixed profile whose unsteady part is a peaked profile. In all cases, the steady model overestimated the power needed by the heating element when compared with the unsteady case. This resulted in underestimates for the $\mathrm{CO}$ of order $0.41 \mathrm{~min}^{-1}$ in the case of the mixed profile $\left(\delta_{M}=0.3\right)$. For the slug flow profile $\left(\delta_{S}=0.95\right)$ this ranged from about $0.41 \mathrm{~min}^{-1}$ at the lower end of the CO scale to upwards of $0.81 \mathrm{~min}^{-1}$ at the higher end. In practice, cardiac output can exceed the upper limits studied here and so we expect the discrepancy to be larger. Therefore, it seems reasonable therefore that when modelling this type of situation one should include the pulsatility of the flood flow.

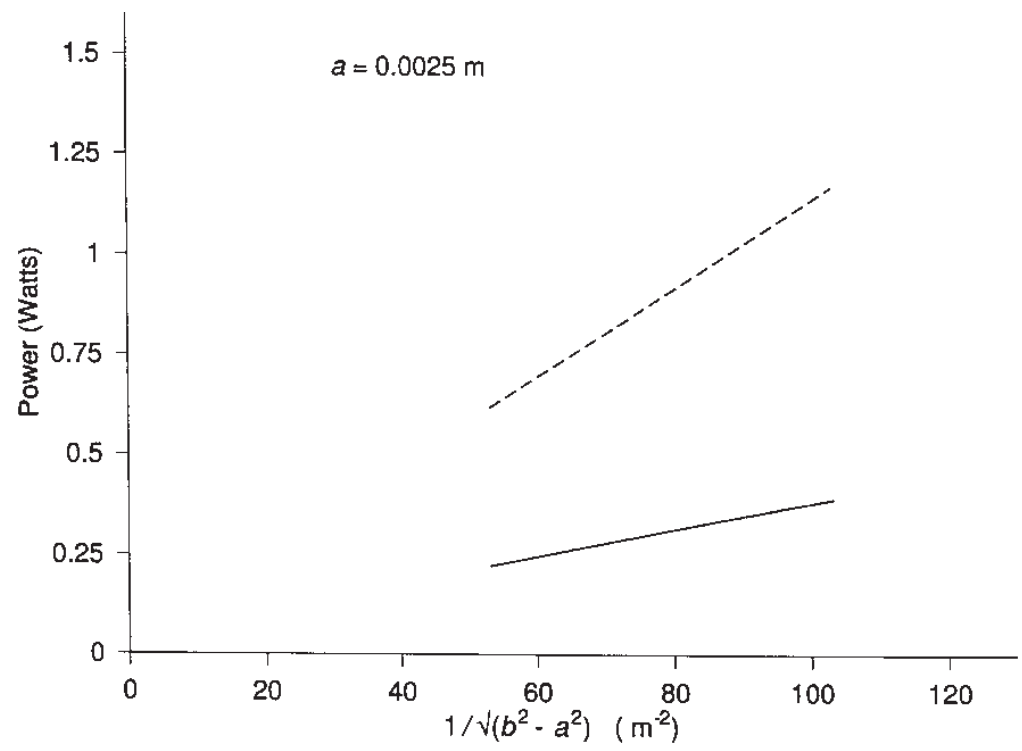

Figure 10. Comparison of power input vs. $1 / \sqrt{\left(b^{2}-a^{2}\right)}$, (a) for a constant CO for fully developed flow (solid line), (b) for constant CO for mixed flow (dashed line). 
The fully developed profile showed the least difference between steady and unsteady models. This may be due to the nature of the steady part of the pulsatile flow being peaked and obeying no-slip boundary conditions. It is interesting to note that this also resulted in a noticeable deviation from the expected behaviour in terms of the relationship between $\mathrm{CO}$ and the power drawn by the heating element. This highlights the calibration problems faced by this sort of technique in assuming that the velocity profile will not change markedly over timescales of the order of minutes. However, inter-patient variability can be suitably reduced through initial in situ calibration with thermodilution measurements.

Of course, we have made many simplifications in our model. These include a rigid cylindrical annulus whose cross-sectional area is well defined thus allowing us to relate velocity measurements to cardiac outputs. This can be a significant drawback in practice for $\mathrm{CO}$ measurements based on HFA techniques. Again the in situ calibration should help to ease these problems. We have also not considered any backflow in our imposed velocity profiles. This would be a realistic addition and is interesting by virtue of the fact that a hot-film anemometer cannot differentiate backflow from forward flow.

\section{Acknowledgements}

Paul Fotheringham and Sandy Gourlay would like to thank the Scottish Higher Education Funding Council for funding this work through RDG grant no.104.

\section{References}

[1] Ehlers, K.C., Mylrea, K.C., Waterson, C.K. and Calkins, J.M., 1986, Cardiac output measurements-A review of current techniques and research, Ann. Biomed. Eng., 14, 219-239.

[2] Pinsky, M.R., 2001, A rose by any other name: cardiac output, Crit. Care Med., 29, 2021-2022.

[3] Hamilton, W.F., Moore, J.W., Kinsman, J.M. and Spurling, R.G., 1932, Studies on the circulation, Am. J. Physiol., 99, 534-551.

[4] Profant, M., Vyska, K. and Eckhardt, U., 1978, The StewartHamilton equations and the indicator dilution method, SIAM J. Appl. Math., 34, 666-675.

[5] Jansen, J.R.C., Schreuder, J.J., Punt, K.D., van den Berg, P.C.M. and Alfieri, O., 2001, Mean cardiac output by thermodilution with a single controlled injection, Crit. Care Med., 29, 1868-1873.

[6] Jansen, J.R.C., Johnson, R.W., Yan, J.Y. and Verdouw, P.D., 1997, Near continuous cardiac output by thermodilution, J. Clin. Monitor, 13, 233-239.

[7] Normann, R.A., Johnson, R.W., Messinger, J.E. and Sohrab, B., 1989, A continuous cardiac output computer based on thermodilution principles, Ann. Biomed. Eng., 17, 61-73.

[8] Ehlers, K.C., Mylrea, K.C. and Calkins, J.M., 1987, Investigation of a continuous heating/cooling technique for cardiac output measurement, Ann. Biomed. Eng., 15, 551-565.

[9] Siegel, L.C., Hennessy, M.M. and Pearl, R.G., 1996, Delayed time response of the continuous cardiac output pulmonary artery catheter, Anesth. Analgesia, 83, 1173-1177.

[10] Poli de Figueiredo, L.F., Malbouisson, L.M.S., Varicoda, E.Y., Carmona, M.J., Auler, Jr. J.O. and Rocha e Silva, M., 1999, Thermal filament continuous thermodilution cardiac output delayed response limits its value during acute hemodynamic instability, J. Trauma, 47, 288-293.
[11] Zollner, C., Polasek, J., Kilger, E., Pichler, B., Jaenicke, U., Briegel, J., Vetter, H.O. and Haller, M., 1999, Evaluation of a new continuous thermodilution cardiac output monitor in cardiac surgical patients: a prospective criterion standard study, Crit. Care Med., 27, 293-298.

[12] Boldt, J., Menges, T., Wollbruck, M., Hammerman, H. and Hempelmann, G., 1994, Is continuous cardiac output measurement using thermodilution reliable in the critically ill patient?, Crit. Care Med., 22, 1913-1918.

[13] Lefrant, J-Y., Bruelle, P., Ripart, J., Ibanez, F., Aya, G., Peray, P., Saïssi, G., de La Coussaye, J-E. and Eledjam, J-J., 1995, Cardiac output measurement in critically ill patients: comparison of conventional thermodilution techniques, Can. J. Anaesth., 42, 972-976.

[14] Bruun, H.H., 1996, Hot-film anemometry in liquid flows, Meas. Sci. Technol., 7, 1301-1312.

[15] Paulsen, P.K., 1980, The hot-film anemometer-a method for blood velocity determination I. In vitro comparison with the electromagnetic flowmeter, Eur. Surg. Res., 12, 140-148.

[16] Paulsen, P.K. and Andersen, M., 1981, Continuous registration of blood velocity and cardiac output with a hot-film anemometer probe, mounted on a Swan-Ganz thermodilution catheter, Eur. Surg. Res., 13, 376-386.

[17] Hasenkam, J.M. and Paulsen, P.K., 1983, Monitoring cardiac output using a special designed velocity catheter and a new cardiac output computer based on hot-film anemometry, Scand. J. Clin. Lab. Investig., 43, 41.

[18] Hasenkam, J.M., Ostergaard, J.H., Pedersen, E.M., Paulsen, P.K. and Nissen, T., 1986, Continuous registration of cardiac-output with a new computer-system designed for hot-film anemometry-an in vitro study, Life Support Syst., 4, 335-344.

[19] Gebhart, B., 1961, Heat Transfer (McGraw-Hill).

[20] Eckert, E.R.G. and Drake, R.M., 1972, Analysis of Heat and Mass Transfer (McGraw-Hill).

[21] Edwards, D.K., Denny, V.E. and Mills, A.F., 1976, Transfer Processes: An Introduction to Diffusion, Convection and Radiation (Hemisphere)

[22] Pedley, T.J., 1980, Fluid Mechanics of Large Blood Vessels (Cambridge University Press).

[23] Chew, M.S., Sloth, E., Veien, M., Jensen, K.V., Kure, H.H. and Hasenkam, J.M., 1996, Movement of pulmonary artery catheters, Heart Vessels, 11, 269-274.

[24] Sømod, L., Hasenkam, J.M., Kim, W.Y., Nygaard, H. and Paulsen, P.K., 1993, 3-dimensional visualization of velocity profiles in the normal porcine pulmonary trunk, Cardiovasc. Res., 27, 291-295.

[25] Sloth, E., Houlind, K.C., Kim, W.Y., Pedersen, E.M., Jorgensen, H.S. and Hasemkam, J.M., 1994, 3-dimensional visualization of velocity profiles in the human main pulmonary-artery with magneticresonance phase-velocity mapping, Am. Heart J., 128, 1130-1138.

[26] Merrill, E.W. and Pelletier, G.A., 1967, Viscosity of human blood: transition from Newtonian to non-Newtonian, J. Appl. Physiol., 23, $178-182$.

[27] Rohlf, K. and Tenti, G., 2001, The role of the Womersley number in pulsatile blood flow-a theoretical study of the Casson model, J. Biomech., 34, 141-148.

[28] MacDonald, D.A., 1982, Fully developed incompressible flow between non-coaxial circular cylinders, J. Appl. Math. Phys., 33, $737-751$.

[29] Womersley, J.R., 1955a, Oscillatory motion of viscous liquid in a thin-walled tube: I. The linear approximation for long waves, Phil. Mag., 46(7), 199-221.

[30] Womersley, J.R., 1955b, Method for the calculation of velocity, rate of flow and viscous drag in arteries when the pressure gradient is known, J. Physiol., 127, 553-563.

[31] Peacock, J., Jones, T., Tock, C. and Lutz, R., 1998, The onset of turbulence in physiological pulsatile flow in a straight tube, Exp. Fluids, 24, 1-9.

[32] Gabe, I.T., Gault, J.H., Ross, Jr. J., Mason, D.T., Mills, C.J., Schillingford, J.P. and Braunwald, E., 1969, Measurement of instantaneous blood flow velocity and pressure in conscious man with a catheter-tip velocity probe, Circulation, 40, 603-614.

[33] McKee, S., Wall, D.P. and Wilson, S.K., 1996, An alternating direction implicit scheme for parabolic equations with mixed derivative and convective terms, J. Comput. Phys., 126, 64-76.

[34] Polhausen, E., 1921, Zur näherungsweisen Integration der Differentialgleichung der laminaren Grenzschischt, Z Angew. Math. Mech., 1, 115. 


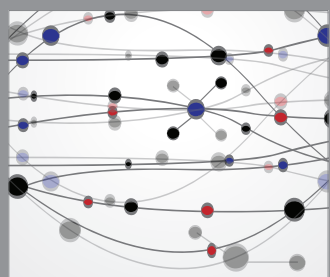

The Scientific World Journal
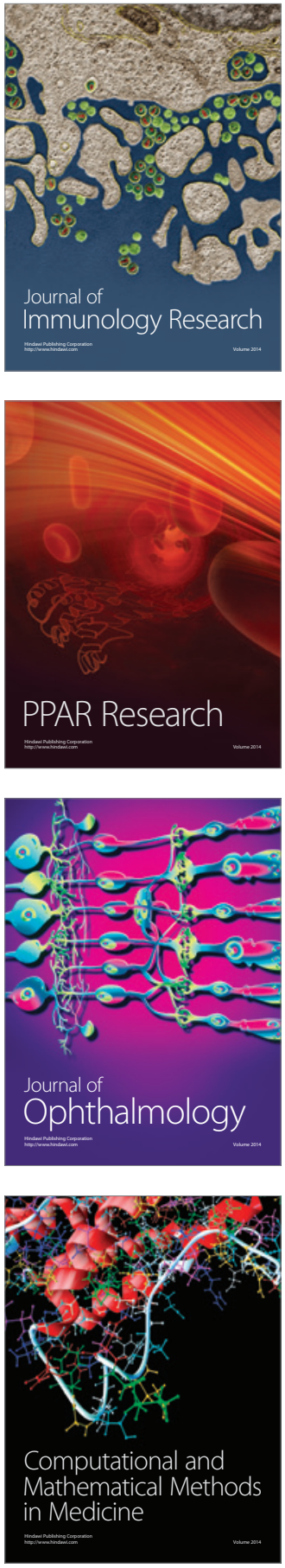

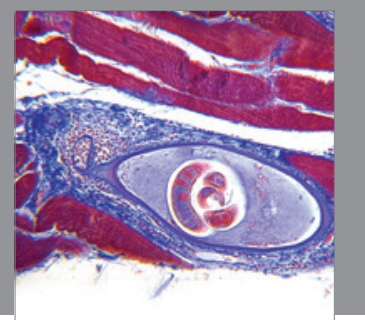

Gastroenterology

Research and Practice
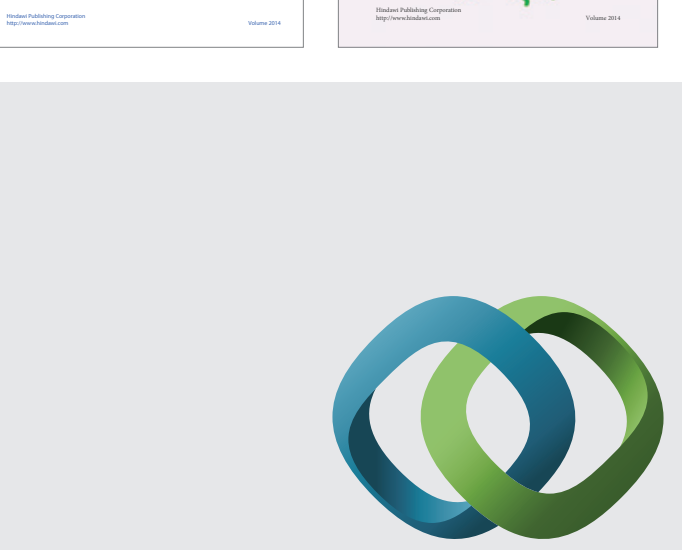

\section{Hindawi}

Submit your manuscripts at

http://www.hindawi.com
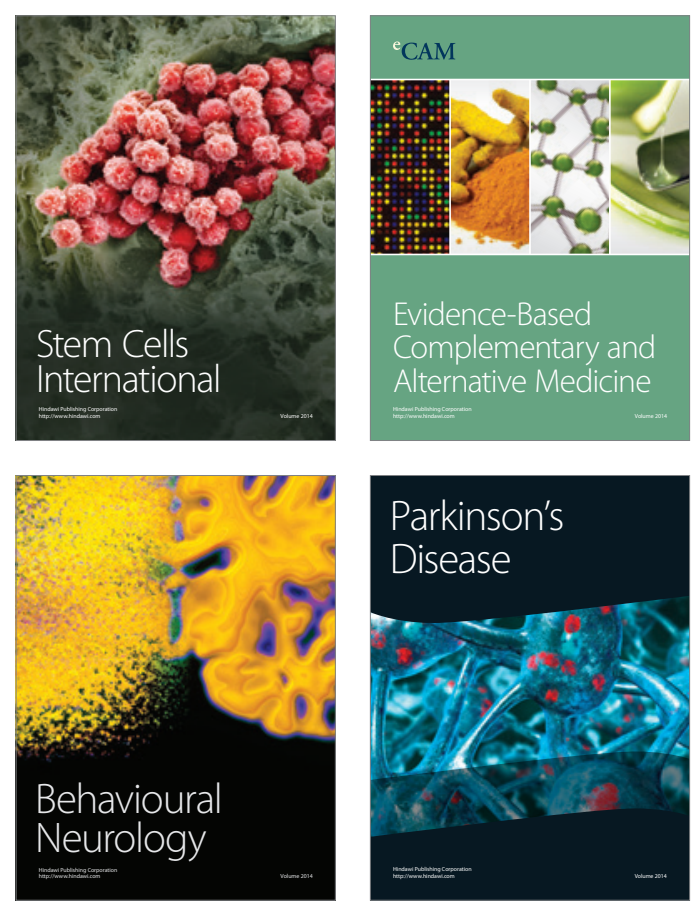

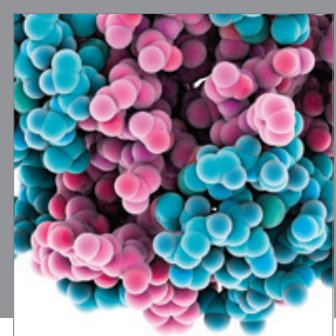

Journal of
Diabetes Research

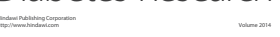

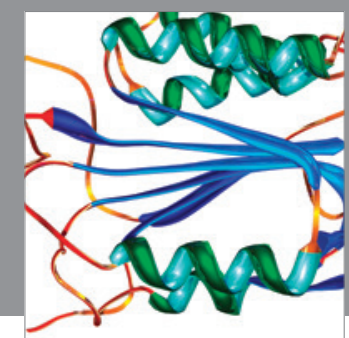

Disease Markers
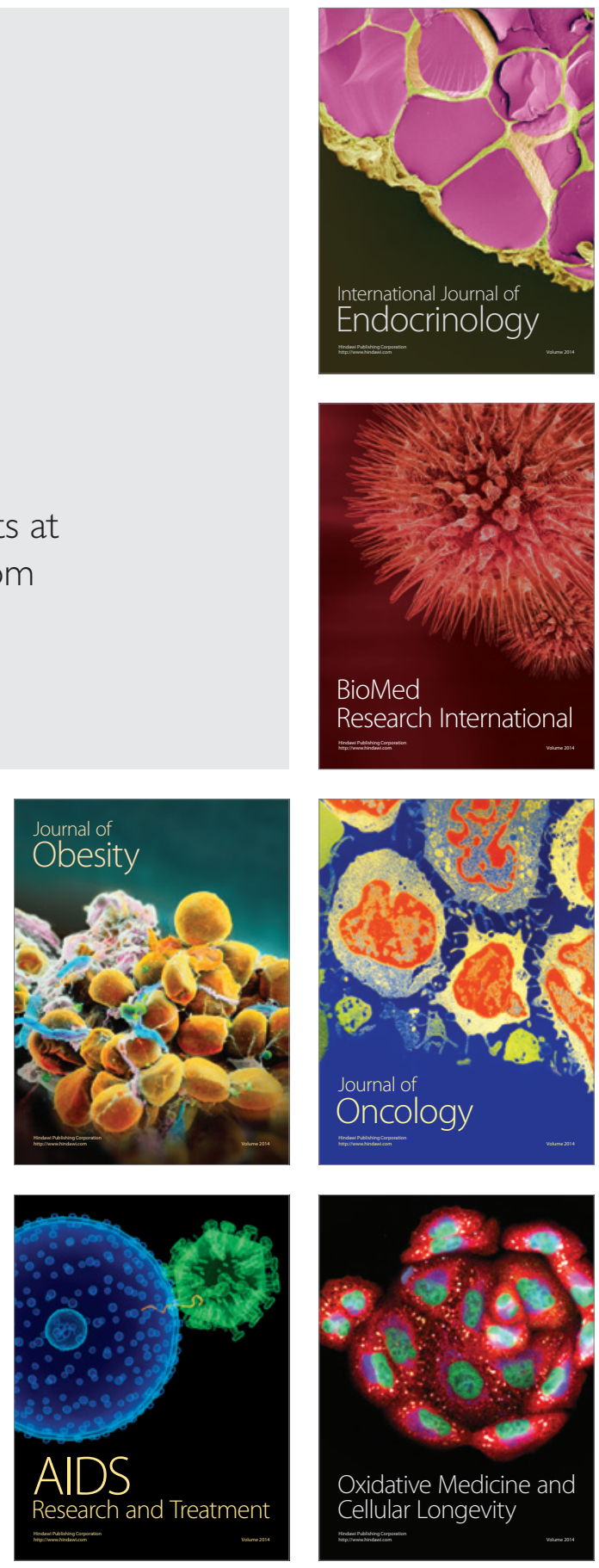